\title{
Más allá de la sostenibilidad: por una Educación Ambiental que incremente la resiliencia de la población ante el decrecimiento
}

\author{
J. Eduardo García Díaz \\ Calle Pirotecnia, s/n, 41013 Sevilla: Departamento Didáctica Ciencias Experimentales y \\ Sociales, Facultad Ciencias Educación, Universidad de Sevilla. Sevilla. España. \\ jeduardo@us.es \\ ORCID: https://orcid.org/0000-0001-7770-9374
}

\section{Jorge Fernández Arroyo}

Calle Pirotecnia, s/n, 41013 Sevilla: Departamento Didáctica Ciencias Experimentales y Sociales, Facultad Ciencias Educación, Universidad de Sevilla. Sevilla. España.

$$
\begin{gathered}
\text { jferarr10@us.es } \\
\text { ORCID: https://orcid.org/0000-0002-5260-9729 }
\end{gathered}
$$

Fátima Rodríguez Marín

Calle Pirotecnia, s/n, 41013 Sevilla: Departamento Didáctica Ciencias Experimentales y Sociales, Facultad Ciencias Educación, Universidad de Sevilla. Sevilla. España.

frodmar@us.es

ORCID: https://orcid.org/0000-0003-0771-6944

\section{María Puig Gutiérrez}

Calle Pirotecnia, s/n, 41013 Sevilla: Departamento Didáctica Ciencias Experimentales y Sociales, Facultad Ciencias Educación, Universidad de Sevilla. Sevilla. España. mpuig@us.es ORCID: https://orcid.org/0000-0002-7536-2976

[Recibido: 30 Noviembre 2018. Revisado: 11 Enero 2019. Aceptado: 15 Enero 2019]

Resumen: En el presente artículo abordamos la problemática asociada al uso del concepto de sostenibilidad en Educación Ambiental. Se presenta una crítica razonada en relación con el contexto actual de decrecimiento/colapso. Apostamos por una educación en y para el decrecimiento como alternativa a la educación para la sostenibilidad.

Palabras clave: Educación Ambiental; Sostenibilidad; Decrecimiento.

Beyond sustainability: for an Environmental Education that increases the resilience of the population in the face of degrowth/collapse

Abstract: In this paper we address the problems associated with the use of the concept of sustainability in Environmental Education. A reasoned critique is presented in relation to the current context of degrowth/collapse. We bet for an education in and for degrowth as an alternative to education for sustainability.

Keywords: Environmental Education; Sustainability; Degrowth. 
Para citar este artículo: García Díaz, J. E., Fernández-Arroyo, J., Rodríguez-Marín, F. y Puig Gutiérrez, M. (2019). Más allá de la sostenibilidad: por una educación ambiental que incremente la resiliencia de la población ante el decrecimiento/colapso. Revista de Educación Ambiental y Sostenibilidad 1(1), 1101. doi: 10.25267/Rev_educ_ambient_sostenibilidad.2019.v1.i1.1101

\section{Introducción}

Parafraseando la idea de Holmgren (2013) en su libro Permacultura: principios y senderos más allá de la sustentabilidad, donde se propone un modelo agroecológico para enfrentar el reto del decrecimiento, proponemos una Educación Ambiental (EA en lo sucesivo), centrada en la perspectiva decrecentista, que vaya más allá de la educación para la sostenibilidad. Pretendemos, por tanto, abrir un debate sobre algunas cuestiones que nos parecen claves para el devenir futuro de la Educación Ambiental.

\section{El problema de la sostenibilidad como principio organizador de la práctica educativa}

Actualmente cualquier educador/a ambiental se encuentra con una gran diversidad de perspectivas dentro del ámbito de la EA: educación para el desarrollo sostenible (EDS), educación para la sostenibilidad (ES), educación ambiental crítica, educación ecosocial, educación para el buen vivir, educación en y para el decrecimiento, etc. Perspectivas que se organizan en torno a un conjunto de tópicos que actúan como elementos centrales estructuradores de cada cuerpo de conocimiento. Por ejemplo, en la EDS el concepto de sostenibilidad es un tópico organizador; en la educación ecosocial lo son las nociones de ecodependencia, interdependencia o la sostenibilidad de la vida; en la educación decrecentista los conceptos de límites biofísicos, decrecimiento y colapso.

Evidentemente, tal diversidad teórica debe trasladarse a la programación y al desarrollo de prácticas educativas concretas que faciliten el cambio de la mentalidad y de la conducta de la población. Pero no resulta fácil plasmar los grandes principios en forma de contenidos educativos, si no se realiza, previamente, un debate profundo sobre el significado de los tópicos que fundamentan y nuclean las diferentes perspectivas de la EA. Más aún, si lo que queremos es plasmar nuestros fundamentos teóricos en forma de modelos didácticos, es decir, modelos que integren el qué enseñar con el cómo enseñarlo y la evaluación (García Pérez, 2000), y que posibiliten propuestas de actuación concretas.

Al respecto, pensamos que el tópico predominante en el panorama actual de la EA, la noción de sostenibilidad, debe cuestionarse como referente para la formulación de contenidos y como organizador de la práctica educativa.

Evidentemente se trata de un concepto polisémico, no existiendo un consenso sobre su significado. En todo caso, en la sostenibilidad entendida como EDS hay un cierto consenso en asociar el desarrollo económico a la conservación del patrimonio cultural y natural, y a la calidad de vida para la humanidad actual y futura, con una doble exigencia ética, de preservación y cuidado de unos recursos naturales que son finitos y de equidad y justicia social (Caride y Meira, 2001). Se pretende conseguir un equilibrio entre los factores económico, ecológico y social (Colom, 2000), con dos claros ejes de referencia: orientar los valores y los comportamientos hacia una 
relación armónica con la naturaleza y redistribuir mejor los recursos planetarios (Novo, 2009). No se pretende limitar el desarrollo (entendido como crecimiento), pero sí vincularlo a la mejora de nuestra relación con el resto del planeta reformando el modelo socio-económico caracterizado por el crecimiento ilimitado, dentro de las coordenadas propias del sistema. Se propone un capitalismo más "humano", con un crecimiento económico ajustado a los condicionantes ecológicos (incluso se propone un crecimiento cero del Producto Interior Bruto (PIB) para los países desarrollados), con una mejor distribución de los recursos (menor desigualdad) y todo ello compatible con la conservación del medio. Discurso bastante ambiguo, pues al mismo tiempo que se admite que hay límites ecológicos al crecimiento también se acepta que hay que mantenerlo (Luffiego y Rabadán, 2000).

Pensamos que son dos las cuestiones claves a debatir: la primera, si la sostenibilidad plantea o no una reforma dentro de las coordenadas del sistema capitalista aceptando su continuidad; y la segunda, si es compatible la idea de sostenibilidad con los datos actuales sobre la situación del mundo (decrecimiento y posible colapso civilizatorio, huida hacia delante de un neoliberalismo cada vez más destructivo).

En relación con el primer tema, se critica que no haya un posicionamiento político claro, al no cuestionar las bases económicas del modelo social basado en el crecimiento, tal como señalan Girault y Sauvé (2008). El problema es que los partidarios de la EDS, aunque insisten en la necesidad y urgencia de transformar las sociedades actuales hacia otros modelos sociales, no terminan por definir su posición ante el capitalismo (Novo, 1998; Vilches, Gil y Cañal, 2010).

Creemos que la rápida popularización y asimilación del concepto se debe, en gran medida, a la necesidad institucional de dar un contenido al modelo de desarrollo deseable que fuera neutro -desde el punto de vista político e ideológico-, pues cualquiera puede apuntarse a la idea de una revolución humanitaria si eso no obliga a optar claramente por unos determinados modelos de organización política y socioeconómica (García, 2004a). Se trata de una mezcla de discurso ético radical con recomendaciones sin operatividad práctica, o que se focalizan, de forma posibilista y pragmática, en ciertas reformas económicas -sin cambios políticos- y en la intervención educativa, aspectos, ambos, bastante asumibles por el sistema dominante. El hecho es que las actuaciones emprendidas bajo el paraguas de la sostenibilidad han tenido poco éxito como instrumento del cambio social (Naredo, 2006), quedando en evidencia que el capitalismo no está realmente interesado en que el tema de la sostenibilidad sea algo más que un mero discurso. En ese sentido, deberíamos reflexionar sobre el argumento que muy atinadamente nos plantea Rull (2010) en relación con el colectivo que tiene mayor poder de decisión: debemos ser conscientes que la negociación con los promotores del modelo de crecimiento ilimitado no ha tenido hasta ahora ningún éxito. En definitiva, es más fácil, y políticamente "más correcto", identificar el sentido del cambio con "ir hacia el desarrollo sostenible", que decir, por ejemplo, que hay que acabar con el actual capitalismo depredador o asumir que las condiciones del mundo están cambiando radicalmente. El punto débil del modelo estaría, pues, en su dimensión política.

El segundo tema está ligado al primero: la decadencia del sistema capitalista va ligada al decrecimiento. En nuestra opinión, el discurso institucional bien intencionado de los objetivos de desarrollo sostenible (ONU, 2015) y de la EDS enmascara la gravedad 
de la crisis al ignorar el hecho de un decrecimiento inevitable, obviando el previsible agotamiento en la primera mitad del siglo XXI de multitud de recursos energéticos y materiales; la inminente llegada del punto de no retorno en el cambio climático; la imposibilidad de mantener un crecimiento exponencial de la población y del uso de los recursos, que tiende matemáticamente a infinito, en un planeta que es finito y en contra de las leyes de la termodinámica y de la ecología. Al respecto, es muy significativo que en los ODS no haya ni una mención al tema del próximo agotamiento de la energía fósil (objetivo 7) y tampoco al carácter destructivo del modelo agrícola industrial y al desastre alimentario asociado al control del tema de la seguridad y soberanía alimentaria por parte de las multinacionales (objetivo 2). En definitiva, los ODS no suponen un cambio apreciable respecto a las propuestas del desarrollo sostenible tradicionales (Suárez-López, Eugenio, Lara y Molina-Motos, 2018), pues se sigue planteando que los graves problemas socio-ambientales actuales pueden ser resueltos dentro del marco del neoliberalismo y del paradigma del crecimiento y la innovación tecnológica.

A pesar de las críticas recibidas, la noción de sostenibilidad sobrevive bajo otras formulaciones. Al respecto, se describe tanto una sostenibilidad "débil" (en la que no hay incompatibilidad entre la continuidad del crecimiento y la naturaleza) representada por la ES institucional, como otra "fuerte" en la que se plantea que un sistema social es sostenible si se ajusta bien, en su interacción con el resto del planeta, al funcionamiento de la naturaleza (Luffiego y Rabadán, 2000; Caride y Meira, 2001). Se adopta en este caso una perspectiva sistémica y más próxima a los conceptos ecológicos: el desarrollo sería sostenible mientras que no rebase la capacidad de carga de los ecosistemas que lo sustentan (Riechmann, 1995). En nuestra opinión, el problema de la sostenibilidad "fuerte" es que era un concepto útil en los años noventa, pero con los datos actuales ya sabemos que cualquier tipo de crecimiento es imposible pues ahora se trata de un ajuste a unos recursos decrecientes (que son los que determinan la capacidad de carga) de forma que ya no hay nada que mantener, sino más bien resistir y evitar la extinción del mayor número posible de seres humanos.

Otra formulación alternativa de la sostenibilidad, es la de la perspectiva ecocéntrica, que está presente en la educación ecosocial, donde se interpreta la sostenibilidad como sostenibilidad de la vida (Gutiérrez, 2018; Herrero, 2018a). Frente a la EDS que propone mantener el desarrollo (se supone que en un sistema capitalista "mejorado") lo que se plantea ahora es mantener la trama de la vida.

Contrastemos este planteamiento con lo que nos dice la epistemología de la ecología. Constatamos que cuando se habla de trama de la vida, no se aclara bien a qué nivel de organización de la materia se hace referencia en cada momento del discurso (células, organismos individuales, poblaciones, biocenosis, ecosistemas, eco-socio-sistemas, biosfera...), asunto clave, pues, en cada nivel, la "vida" presenta características emergentes diferentes (Morin, 1986 y 1987). Y si decimos "debemos cuidar la vida" ¿en todos esos niveles tiene sentido la frase? ¿es indiscutible que la vida tiene un valor intrínseco? ¿qué significa, por ejemplo, cuidar los ecosistemas o que los ecosistemas se cuiden entre sí? ¿tiene sentido decir que un lago y un bosque, situados en espacios adyacentes, se "ayudan mutuamente" o tienen "su propia ética"? El fondo de la cuestión es si realmente estamos adoptando una perspectiva compleja (en el sentido de Morin) o más bien un enfoque reduccionista del tema, en el que se acepta, 
sin más, una interpretación de la vida que, como veremos a continuación, es muy discutible.

La educación ecosocial interpreta la trama de la vida como un "superorganismo" (De Castro, 2008 y 2013), que se autoorganiza (Herrero, 2018b). Estos enunciados se corresponden con la perspectiva del holismo organicista, perspectiva muy discutida desde la epistemología de la ecología (O’Neill, De Angelis, Waide y Allen, 1986; Drouin, 1984 y 1988; Acot, 1990; Deléage, 1992 y 1993; Golley, 1993). Estos autores consideran que el holismo organicista es una concepción reduccionista e idealista (frente a un holismo materialista). Su argumento central sería que organismos y ecosistemas tienen propiedades emergentes diferentes. Los organismos tienen como características básicas una organización jerárquica y centralizada, y cuya estabilidad oscila en torno a un óptimo preestablecido, siendo muy relevantes los bucles recursivos y el cambio cíclico, propios de un proceso de autoorganización (Morin, 1987). Están "equilibrados" siempre que nos movamos en torno a ese estado óptimo. Al tener un intervalo de variación corto (por ejemplo, no podemos alejarnos demasiado de niveles como el de la glucemia si queremos evitar la desorganización del sistema) los organismos son extremadamente vulnerables (enferman, mueren, se desorganizan con facilidad), lo que justifica el cuidado. Cuidados que se dan tanto en las relaciones intraespecíficas (supervivencia de poblaciones y especies) como en las interespecíficas (relaciones de complementariedad, como la simbiosis, que forman parte de las redes tróficas). Sin embargo, en un ecosistema encontramos una organización en red horizontal, descentralizada, y no existe, además, un estado óptimo preestablecido, ya que el ecosistema presenta un equilibrio dinámico (en cada reequilibración el sistema cambia de forma irreversible) evolucionando a lo largo del tiempo por reorganización (Morin, 1987).

Por tanto, considerar el ecosistema como un sistema similar al de un organismo individual, pensamos que es una reducción mecanicista, incompatible con la descripción del ecosistema que nos hace la ecología como un sistema con sus propias propiedades emergentes singulares (Levins y Lewontin, 1980; Margalef, 1980; McIntosh, 1985; Morin, 1987; Deléage, 1993; Golley, 1993) entre las que destaca la de ser un sistema abierto, que como entidad histórica ha utilizado el flujo de energía para reorganizarse continuamente, organización (entendida como información) que, a su vez, condiciona la circulación de materiales y el flujo de energía en nuestro planeta (Margalef, 1980). No es apropiado, por tanto, atribuir a los ecosistemas cambios cíclicos (oscilación en torno a un óptimo), sino hablar más bien de un cambio helicoidal (Margalef, 1974), con una componente "cíclica" y con otra, mucho más determinante, irreversible, de carácter evolutivo.

Estos argumentos nos identifican con la opinión de Rull (2010), cuando sostiene que la sostenibilidad además de ser una concepción antropocéntrica es también una concepción mítica. Y es mítica en cuanto que no se apoya en la concepción científica más aceptada sobre lo que es un ecosistema: sistema abierto en continua reorganización sin un estado óptimo preestablecido, que cambia de forma irreversible. Como nos indica Frank (2018) si adoptamos una perspectiva evolutiva (de millones de años de historia planetaria) comprendemos que la biosfera siempre se reorganiza, incluso después de impactos de la magnitud del actual cambio climático, por lo que, según este autor, el tema a discutir no es salvar la Tierra (no tiene sentido hablar de "salvación" y si queremos usar el término "se salvaría sola") sino como 
salvar a una población humana integrada en una biosfera que va a cambiar fuertemente (como adaptarnos).

En conclusión, creemos que el tópico "sostenibilidad" no se corresponde con una visión "ecocéntrica" que asuma las aportaciones de la ecología, ni se debe asociar con la noción de decrecimiento, cuestión que abordaremos con más detalle a continuación.

\section{¿Dónde poner el foco? El debate sobre la inevitabilidad y el alcance del decrecimiento/colapso}

Es tradicional en EA partir de un diagnóstico de la situación planetaria como paso previo a la formulación de propuestas educativas. Cada corriente de pensamiento de la EA (como la conservacionista, la EA crítica, o la EDS) se asocia a un determinados análisis sobre la situación del mundo.

Todas estas tendencias se originan entre los años setenta y noventa del siglo pasado, recogiendo las distintas percepciones existentes sobre el cambio social y ambiental presentes en ese momento. Pero ante la evolución actual de la situación del mundo ¿podemos seguir manteniendo los postulados de la EA del siglo pasado?

Desde finales del siglo pasado y comienzos del presente siglo, se retoma con fuerza la idea de los límites del crecimiento, ya enunciada en los años setenta (Meadows et al, 1972). Se comienzan a manejar las nociones de "emergencia planetaria" y de "educar para la supervivencia" (Vilches y Gil, 2009). Estas ideas aparecen cada vez con más frecuencia en el discurso "experto", aunque apenas llegan al ámbito de la práctica de educadores y educadoras (Edwars, Gil, Vilches y Praia, 2004).

Por otro lado, y vinculada al movimiento ecosocial, aparece una amplia literatura sobre los conceptos de límites biofísicos (básicamente el agotamiento de los recursos materiales y energéticos y el cambio climático), el decrecimiento y posible colapso civilizatorio (Acosta y Ulrich, 2018; Casal Lodeiro, 2016; Fernández y González, 2014; Holmgren, 2013; Latouche, 2007, 2009 y 2012; Prats, Herrero y Torrego, 2016; Taibo, 2016; Trainer, 2017, Turiel, 2011, 2016 y 2017). Dentro de este movimiento habría dos corrientes de pensamiento: una corriente más reformista próxima a la obra de Latouche (aún es posible evitar el choque mediante un decrecimiento voluntario) y otra más radical que plantea un decrecimiento obligado e inevitable (RodríguezMarín, Fernández-Arroyo y García-Díaz, 2015; García, Navarro, de Alba y del Carmen, 2017).

En nuestra opinión, la perspectiva decrecentista cuestiona la noción de sostenibilidad. Es decir ¿tiene sentido hablar de mantener algo cuando estamos en una situación radicalmente desequilibrante de choque con nuestros límites biofísicos? ¿tiene sentido seguir hablando de conservar nuestro estilo de vida o de conservar la biosfera "tal como es" en vez de comenzar a adaptarnos a una situación radicalmente nueva?

Al respecto, pensamos que la EA está en una encrucijada, en un proceso de transición, en el que se entremezclan los discursos del decrecimiento con el de la sostenibilidad. Ello es evidente en el caso de la sostenibilidad "fuerte" y tendencias derivadas como la educación ecosocial, en la que se asocia el conocido discurso de la sostenibilidad con un nuevo elemento: la necesidad de incrementar la resiliencia de la población (Assadourian, 2017). Para este autor, principios básicos de esta corriente educativa 
serían comprender la ecodependencia de la humanidad respecto de la Tierra (lo asocia a una ecoalfabetización); o la interdependencia entre las personas (fomento de las habilidades sociales, de los cuidados y de las relaciones de complementariedad). Creemos que esos principios son esenciales en EA. El problema es la asociación entre ecoalfabetización con sostenibilidad. Así, Stone (2017) habla de educar para la sostenibilidad, proponiendo la comprensión del funcionamiento de la biosfera y el ajuste de la actividad humana a dicho funcionamiento (ecoalfabetización), aprendiendo de la naturaleza (biomímesis) y convirtiendo la sostenibilidad en una práctica comunitaria asociada a la acción local. Assadourian (2017) también asocia la idea de educar en resiliencia con la de educar en la sostenibilidad, mostrando su confianza en que los gobiernos potencien una educación para la sostenibilidad y la resiliencia aplicando el objetivo 4 de los ODS (para el 2030). Los autores citados, en ningún momento, discuten la pertinencia o no del uso del concepto de sostenibilidad (y sus implicaciones políticas).

Las preguntas claves son ¿por qué mantenemos un término como el de la sostenibilidad si es tan confuso y está tan discutido? ¿por qué asociarlo a términos como ecocentrismo, decrecimiento o resiliencia? ¿por qué, en el caso de los partidarios de una sostenibilidad "fuerte", no abandonamos ya el término y hablamos sin rodeos de educar en y para el decrecimiento? En nuestra opinión, la EA debe "liberarse" y "descontaminarse" del discurso de la sostenibilidad, pues creemos que el concepto se mantiene no por sus bondades como principio organizador de un campo teórico sino por la fuerte presión institucional existente sobre los colectivos correspondientes. Ante esa presión, si utilizamos "instrumentalmente" los programas institucionales para cubrir nuestros objetivos, no deberíamos asumir, sin más, su relato, que deviene así en una forma de pensamiento único.

En el fondo, aunque todas y todos reconocemos la importancia de la problemática socioambiental, no está nada claro si la crisis sistémica supone o no un cambio civilizatorio radical, y si debemos centrar nuestro esfuerzo en reformar el sistema (parchearlo) en el sentido de la EDS o en preparar a la población para que el inevitable choque sea lo menos lesivo posible, pues no es lo mismo capacitar a las futuras generaciones para tratar con los problemas sociales y ambientales en un contexto similar al actual (planteamiento que identificamos con la EDS) que hacerlo para un contexto caracterizado por un mundo de baja energía, con menos recursos de todo tipo, transformado radicalmente por el cambio climático y liderado por un capitalismo destructor. Proponemos abandonar el término o, en todo caso, si hablamos de sostenibilidad, referirla a la supervivencia de nuestra especie, desarrollando una respuesta colectiva ante un cambio planetario tan fuerte. Debatir esta cuestión ayudaría a clarificar nuestros planteamientos teóricos y al enunciado de contenidos educativos concretos.

Aunque nuestro enfoque asume muchos de los principios de la educación ecosocial (González, 2018; Gutiérrez, 2018; Herrero, 2018a), es decir, propuestas como la ecoalfabetización, la necesaria interdependencia entre los seres humanos (cuidados), la importancia de la vinculación al territorio próximo, la relevancia de la diversidad, de la creatividad, o la ética de la complementariedad; somos críticos con mantener el término sostenibilidad (que consideramos incompatible con decrecimiento $\mathrm{y}$ ecocentrismo). También compartimos la idea de priorizar aquellos contenidos que 
ayuden a un decrecimiento justo y ordenado, tema al que dedicaremos el siguiente apartado.

\section{La formulación de contenidos desde una perspectiva decrecentista}

En el caso del qué enseñar, habría que realizar una integración didáctica de distintos referentes a la hora de formular y organizar los contenidos (García, 2004a y 2004b), referentes tales como un determinado diagnóstico de la situación del mundo (nuestra percepción sobre los problemas socioambientales o la crisis sistémica), las concepciones de las personas que aprenden -próximas al conocimiento cotidiano-, y las aportaciones de los conocimientos organizados -sistemas ideológicos, conocimiento científico-. En otros términos, si queremos elaborar en EA itinerarios didácticos para una construcción gradual y progresiva del contenido (RodríguezMarín, Fernández-Arroyo y García Díaz, 2014; García, Porlán y Navarro, 2017), deberíamos reflexionar tanto sobre la formulación del contenido en el punto de partida del proceso enseñanza-aprendizaje (por ejemplo, las concepciones sobre la "vida", la "sostenibilidad" o el "decrecimiento" presentes en el conocimiento cotidiano y las barreras para el cambio asociadas a dichas concepciones) como sobre su formulación en el punto de llegada deseable (qué significa, por ejemplo, que lo ideal es "sostener la vida", "mantener el crecimiento en armonía con el resto de la biosfera" o "incrementar la resiliencia de la población").

En una EA centrada en el decrecimiento el criterio básico debe ser fomentar el ajuste a la nueva situación del mundo. Dicho ajuste supone desarrollar respuestas educativas a tres retos. El primer reto es la disminución de los recursos, asociada además a una posible crisis institucional que exija respuestas locales. Este reto implica educar en la autonomía y en la autosuficiencia, y más concretamente en un "saber hacer" basado en el manejo de tecnologías más resilientes. Un segundo reto tiene que ver con el incremento de la incertidumbre y la vulnerabilidad. Ante problemas complejos y novedosos y situaciones de mayor riesgo necesitamos potenciar una comprensión sistémica del mundo, la capacidad investigadora (y por ende la creatividad), el trabajo colaborativo y los cuidados. Es notable en EA la dispersión de propuestas metodológicas que impide definir con claridad con qué modelo didáctico estamos trabajando, lo que no deja de ser una manifestación de activismo (Rodríguez y García, 2009). Proponemos una metodología didáctica basada en la investigación, de corte constructivista, que capacite a las personas para resolver los problemas asociados a la situación de decrecimiento, metodología que integraría propuestas metodológicas más concretas, evitando una dispersión metodológica que dificulta que nuestra intervención educativa sea coherente (García, 2004a; García y Cano, 2006). El tercer reto es la rapidez del cambio (en apenas unas decenas de años podríamos encontrarnos en una situación muy diferente). No habrá mucho tiempo para el ensayo-error, para experimentar las posibles respuestas al decrecimiento/colapso, por lo que es prioritario educar en el espíritu crítico, en el incremento de nuestra capacidad evaluadora, y en una ecoalfebetización basada en el conocimiento científico más que en el pensamiento mítico.

Planteamos un cambio radical en los contenidos, revisando el significado de los contenidos curriculares tradicionales en clave decrecentista (reformularlos de manera que su tratamiento sea útil para comprender el choque con nuestros límites 
biofísicos y para capacitar a la gente para afrontarlo) y priorizando el desarrollo de determinados contenidos. No significa por tanto crear nuevos contenidos pero sí reformular la finalidad con la que se trabajan esos contenidos, ya que la visión tradicional no responde a los retos del decrecimiento.

En cuanto a los contenidos prioritarios, parece claro que lo serían todos aquellos que ayuden a capacitar a la población para adaptarse al decrecimiento. Ejemplo de ello serían nociones como: eco-socio-sistema, ciclos y flujos de energía en la biosfera, agotamiento de recursos, límites biofísicos, pico del petróleo, tasa de retorno energético, huella ecológica, factor limitante, metabolismo social, cambio climático, modelos agroecológicos y seguridad alimentaria, entre otras.

Veamos ahora algunos ejemplos concretos, relativos al cambio de perspectiva que proponemos, en relación con algunos tópicos prioritarios (García, Rodríguez-Marín, Fernández-Arroyo y Puig, 2019).

En la literatura de la EA hay un claro consenso en que hay que trabajar con sistemas. El problema es que este enfoque choca con la compartimentación del saber presente tanto en la formación del profesional de la EA como en las propuestas curriculares institucionales. Por eso es prioritario romper con esa atomización, superando la disociación entre lo natural y lo social, y trabajando con una visión más global e integrada en el sentido del paradigma de la complejidad de Edgar Morin (1986, 1987, 1988, 1992, 1994 y 2001). Tendríamos que trabajar con eco-socio-sistemas (Morin, 1987), en los que resulta básico comprender el funcionamiento de la circulación de materiales y del flujo de energía planetarios, y como la actividad humana está integrada en esos ciclos y flujos, dependiendo de ellos (ecodependencia).

Se trataría de sustituir el enfoque atomizado, enciclopédico, aditivo, reduccionista y simplificador, centrado en el ecosistema o en el sistema social como una mera suma de elementos y en la disociación entre los mismos, superando la idea mecanicista de las relaciones causales lineales (causa-efecto) mediante la construcción de la noción de interacción (García, 2001) y de redes (redes tróficas, redes sociales, diferenciación entre organizaciones piramidales y organizaciones horizontales en función de su resiliencia, etc.); centrándonos más en las interacciones que suponen complementariedad (simbiosis, cooperación, solidaridad) por su papel determinante para la organización del eco-socio-sistema, que en las relaciones de antagonismo (depredación, competencia, lucha de todos contra todos), que son las más populares en nuestro acervo cultural.

Este cambio de perspectiva implica otra forma de trabajar la materia y la energía, dando prioridad a la investigación de los ciclos de la materia y el flujo de energía en nuestro planeta, evitando, de nuevo, una visión atomizada y parcial del conocimiento (la materia y la energía se suelen estudiar como asuntos ajenos al funcionamiento del eco-socio-sistema, sin una visión de conjunto). Se trata de ir más allá de la cadena causal que conecta la producción con el consumo y la emisión de residuos, ubicando todo el proceso en el marco del funcionamiento de la biosfera. Aquí, el tema del agotamiento de los recursos en relación con los ciclos de la materia y los flujos de energía y el tema del cambio climático asociado a las emisiones de gases de efecto invernadero serían temas básicos para comprender el decrecimiento. En relación con estos dos temas, resulta esencial superar las barreras para el cambio asociadas a nuestros estereotipos culturales (confianza ilimitada en la solución tecnológica, 
negacionsimos, etc.) En relación con el cambio climático, los más conocidos son la confusión existente entre cambio climático y el deterioro de la capa de ozono (el 67 \% de la gente), o la confusión entre clima y tiempo atmosférico. Pero hay muchas claves del tema que pasan desapercibidas (no solo en los medios de comunicación sino también en algunos documentos ecologistas y de educación ambiental): la relevancia del metano como gas de efecto invernadero (que se desconoce en general); la relación entre cambio climático y modelo energético (en los materiales didácticos existentes casi no aparece la relación entre cambio climático y capitalismo y menos aún entre cambio climático y decrecimiento-colapso); el papel de la fotosíntesis, de la respiración y de las combustiones en el ciclo del carbono; el alcance en cuanto a las consecuencias de determinados cambios (por ejemplo, se insiste mucho en temas como los cambios meteorológicos o la subida del nivel del mar, y mucho menos en otros temas aún más relevantes, como el deshielo del permafrost o el cambio en la acidez de las aguas oceánicas); la existencia de procesos de retroalimentación positiva que pueden agravar el problema, etc. El tema del cambio climático y del ciclo del carbono es muy complejo, pues supone comprender un ciclo biogeoquímico, estudiando los posibles itinerarios que sigue el carbono y los procesos implicados (combustión, respiración, fotosíntesis).

Es esencial conectar estos conceptos con los problemas cotidianos que habría que enfrentar en una situación de decrecimiento: cómo obtener agua de un pozo si ya no tengo electricidad; cómo construir un habitáculo fresco en verano y caliente en invierno; quién tiene mayor eficiencia energética, un cultivo industrial o un huerto en permacultura, una cocina eléctrica u otra de gas .... También trabajar nociones claves como la degradación de la energía (concepto básico para entender como no puede haber un crecimiento ilimitado en un sistema finito) o la eficiencia energética (medida como tasa de retorno energético). Al respecto, es fundamental comprender la idea de que para una misma cantidad de energía disponible distintas organizaciones sociales presentan distintas eficiencias. No tiene la misma eficiencia un huerto en permacultura que una explotación agrícola industrial, una organización horizontal de redes locales que una estructura jerarquizada piramidal, una comunidad organizada en viviendas unifamiliares que una organización comunal con servicios compartidos, un transporte de materiales en el que predomina la componente horizontal que otro más vertical que prime el ciclo de nutrientes ...

Las idea de capacidad de carga del ecosistema, de huella ecológica, etc. son nociones complejas por lo que habría que comenzar, en una secuencia didáctica, con el concepto de límite y el de distribución desigual de los recursos en el planeta. Posiblemente sea más fácil comprender los impactos si se trabaja como capacidad de carga del ecosistema en relación con elementos concretos. El fondo del tema es si hay recursos limitados. El problema es que el término "limitados" no se entiende por parte de personas que vivencian la falta de límites en un mundo consumista donde predomina la idea de que los humanos pueden acceder indefinidamente a todo tipo de recursos. Hay que construirlo a partir de itinerarios didácticos montados con experiencias concretas.

Este enfoque supone también otra manera de trabajar la historia de la humanidad. La historia de las sociedades suele hacerse siguiendo un orden cronológico, de forma que se van presentando los acontecimientos más destacados de cada uno de los períodos históricos determinados; cronología que ignora otras culturas y otras 
civilizaciones (eurocentrismo). Se suelen presentar algunas características generales del contexto en el que ocurren los acontecimientos, así como se presta especial atención a las fechas y personajes históricos. No suele haber una aproximación ecológica al tema (por ejemplo, nunca se habla de los cambios en el metabolismo social o de la relación entre tecnología y recursos) ni se estudia como distintas civilizaciones enfrentan el problema de los recursos y de los residuos. Podríamos seleccionar algunos de los conflictos actuales e indagar acerca de su origen y sus causas. Esto nos llevará en la mayoría de las ocasiones a conectar con los recursos naturales y la forma en la que se articula el poder en base a su dominio. Conectar la realidad actual con el pasado permite desarrollar un pensamiento crítico y reflexivo apoyado en argumentos. A su vez, analizar los problemas más relevantes del presente y establecer relaciones entre estos y la interacción humanidad-planeta ofrece una visión sistémica de la historia, que sería clave desde una perspectiva decrecentista. Al respecto, creemos que habría que investigar los tres modelos de organización social más determinantes en dicha historia: las sociedades recolectoras, las agrícolas y las industriales, siempre en relación con la circulación de materiales y el flujo de energía (metabolismo social), tal como proponen Fernández y González (2014).

Pero, sobre todo, en una situación de decrecimiento, necesitamos respuestas al reto de asegurar la alimentación humana en un mundo de baja energía y con un rápido cambio de la biosfera asociado al cambio climático. Este hecho nos lleva a un asunto educativo clave: hay que desarrollar el saber hacer en relación con la producción de alimentos. Es cierto que en EA se utiliza el huerto como recurso, pero lo que planteamos es, de nuevo, un cambio de perspectiva: primero, el huerto debe ser un espacio de entrenamiento para incrementar nuestra resiliencia más que un mero divertimento; segundo, no nos vale cualquier huerto, sino aquél que mejor nos ajuste al cambio ecológico en curso (Rodríguez-Marín, Fernández-Arroyo y García, 2015). Podemos tener un huerto y utilizarlo para que se comprenda cómo crecen y se desarrollan las plantas hortícolas o podemos utilizarlo para trabajar factores limitantes (nutrientes del suelo, agua, luz ...), y para que se reconozca la relación entre organización social y eficiencia en el uso de los recursos, tomando decisiones organizativas (diseño del huerto, disposición y preparación de los bancales, sistemas de riego, reciclaje y compostaje, uso o no de acolchado, de plantas complementarias y de bosque de alimentos, etc.) y relacionándolas con la producción del huerto. Al respecto, es imprescindible introducir modelos agroecológicos del tipo de la permacultura, que presentan una elevada TRE (tasa de retorno energético que mide la eficiencia energética de un sistema) y un gran ahorro de recursos por su alto grado de ajuste a los ciclos naturales potenciando el transporte vertical de materiales sobre el horizontal. En relación con el tema hay que relacionar suelo y seguridad alimentaria. Por ejemplo, un lugar común en nuestros centros educativos es relacionar la alimentación de las plantas con en el proceso de obtención de glucosa. Un cambio de perspectiva de la fotosíntesis sería trabajar más detenidamente qué ocurre con los nutrientes del suelo.

Por último, señalar que para trabajar los contenidos desde una perspectiva decrecentista resulta imprescindible asociar la comprensión con los valores, la emoción y la acción, vinculando el aprendizaje con el "saber hacer", el compromiso político y la intervención social, educando en el desarrollo práctico de proyectos concretos (huertos ecológicos sociales y escolares, reforestación de enclaves, defensa 
de una zona verde o del arbolado de un lugar, mercadillos ecológicos, reciclaje de residuos, participación en campañas de comunicación o contra la instalación y mantenimiento de determinadas industrias ...).

\section{Referencias}

Acosta, A. y Ulrich, B. (2018). Salidas del laberinto capitalista. Decrecimiento y postextractivismo. Barcelona: Icaria Editorial.

Acot, P. (1990). Historia de la ecología. Madrid: Taurus.

Assadourian, E. (2017). Educación ecosocial: cómo educar frente a la crisis ecológica. En Informe Anual del WorldWatch Institute, Educación ecosocial. pp.25-49. Barcelona: Icaria Editorial.

Caride, J. A. y P. A. Meira (2001). Educación ambiental y desarrollo humano. Barcelona: Ariel.

Casal Lodeiro, M. (2016). La izquierda ante el colapso de la civilización industrial. Madrid: La Oveja Roja.

Colom, A. J. (2000). Desarrollo sostenible y educación para el desarrollo. Barcelona: Octaedro.

De Castro, C. (2008). El origen de Gaia. Una teoría holista de la evolución. Badajoz: @becedario.

De Castro, C. (2013). En defensa de una teoría Gaia orgánica. Ecosistemas, 22(2), 113118.

Deléage, J. P. (1992). Aux origines de la science écologique: a propos de quelques ouvrages récents. Revue D'histoire des Sciences, 45(4), 477-490.

Deléage, J. P. (1993). Historia de la ecología. La ciencia del hombre y de la naturaleza. Barcelona: Icaria Editorial.

Drouin, J. M. (1984). La Naissance du concept d'écosystéme. Thése pour le doctorat présentée L'université Paris I.

Drouin, J. M. (1988). Un éxito reciente. Historia del concepto de ecosistema. En A. Giordan, et al., Conceptos de biología, Tomo 1. Barcelona: Labor.

Ecologistas en Acción (2015). 99 preguntas y 99 experiencias para aprender a vivir en un mundo justo y sostenible. https://www.ecologistasenaccion.org/?p=20300

Edwars, M., Gil, D., Vilches, A. y Praia, J. (2004). La atención a la situación del mundo en la educación científica. Enseñanza de las Ciencias, 22(1), 1-17.

Fernández, R. y González, L. (2014). En la espiral de la energía. Madrid: Libros en Acción. Baladre.

Frank, A. (2018). De hecho ¿qué quiere decir eso de "salvar" a la Tierra?. Rebelión: http://www.rebelion.org/noticia.php?id=243390.

García, J. E. (2001). La construcción de la noción de interacción. Alambique, Didáctica de las Ciencias Experimentales, 27, 92-106.

García, J. E. (2004a). Educación Ambiental, Constructivismo y Complejidad: una propuesta integradora. Sevilla: Díada. 
García, J. E. (2004b). Los contenidos de la educación ambiental: una reflexión desde la perspectiva de la complejidad. Investigación en la Escuela, 53, 31-52.

García, J. E. y M. I. Cano (2006). ¿Cómo nos puede ayudar la perspectiva constructivista a construir conocimiento en Educación Ambiental? Revista Iberoamericana de Educación, 41, 117-131.

García, J. E., Navarro, E., De Alba, N. y Del Carmen, M. (2017). Educar en y para el decrecimiento. Comunicación presentada en el VIII Encuentro Iberoamericano de colectivos y redes de maestros y maestras, educadores y educadoras que hacen investigación e innovación desde la escuela y la comunidad. Morelia, Michoacán, México, del 10 al 21 de Julio de 2017.

García, J. E., Porlán, R. y Navarro, E. (2017). Los fines y los contenidos de la enseñanza. En Enseñanza Universitaria. Como mejorarla. Madrid: Morata.

García, J. E., Rodríguez-Marín, F., Fernández-Arroyo, J. y Puig, M. (2019). La educación científica ante el reto del decrecimiento. Alambique, Didáctica de las Ciencias Experimentales, 95, 47-52.

García, J. E., Rodríguez-Marín, F., Solís, M. C. y Ballenilla, F. (2007). Investigando el problema del uso de la energía. Investigación en la Escuela, 63, 29-45.

García Pérez, F. F. (2000). Los modelos didácticos como instrumento de análisis y de intervención en la realidad educativa. Scripta nova. Revista electrónica de Geografía y Ciencias Sociales, 64. http://www.ub.edu/geocrit/sn-64.htm.

Girault, Y. y Sauvé, L. (2008). L'éducation scientifique, l'éducation à l'environnement et l'éducation pour le développement durable. Aster, 46, 7-30.

Golley, F. B. (1993). A History of the Ecosystem Concept in Ecology: More than the Sum of the Parts. New Haven and London: Yale University Press.

González, L. (coord.) (2018). Educar para la transformación ecosocial. Orientaciones para la incorporación de la dimensión ecosocial al currículo. Madrid: FUHEM.

Gutiérrez, J. M. (2018). Educatio ambientalis. Invitación a la educación ecosocial en el Antropoceno. Bilbao: Bubok.

Herrero, Y. (2018a). Educar para la transformación ecosocial. Conferencia en la Escuela de Verano de FUHEM. https://www.youtube.com/watch? v=jvYGZ6JLNf0

Herrero, Y. (2018b). Organizar la vida en común en el Antropoceno. CTXT, 196 (21/11/2018). https://ctxt.es/es/20181121/Firmas/22927/constitucionantropoceno-ecologismo-yayo-herrero.htm

Holmgren, D. (2013). Permacultura: principios y senderos más allá de la sustentabilidad. Argentina: Kaicron.

Latouche, S. (2007). Sobrevivir al desarrollo. Barcelona: Icaria Editorial.

Latouche, S. (2009). Pequeño tratado del decrecimiento sereno. Barcelona: Icaria Editorial.

Latouche, S. (2012). La sociedad de la abundancia frugal. Barcelona: Icaria Editorial. 
Levins, R. y Lewontin, R. (1980). Dialectics and reductionism in ecology. Synthèse, 43, 47-78.

Luffiego, M. y J. M. Rabadán (2000). La evolución del concepto de sostenibilidad y su introducción en la enseñanza. Enseñanza de las Ciencias, 18(3), 473-486.

Margalef, R. (1974). Ecología. Barcelona: Omega.

Margalef, R. (1980). La biosfera, entre la termodinámica y el juego. Barcelona: Omega.

McIntosh, R. P. (1985). The background of ecology. Cambridge: Cambridge University Press.

Meadows, D. H., Meadows, D. L., Randers, J. y Behrens, W. (1972). Los límites del crecimiento. Madrid: Fondo de Cultura Económica.

Morin, E. (1986). El Método I: La Naturaleza de la Naturaleza. Madrid: Cátedra.

Morin, E. (1987). El Método II: La Vida De la Vida. Madrid: Cátedra.

Morin, E. (1988). El Método III: El Conocimiento del Conocimiento. Madrid: Cátedra.

Morin, E. (1992). El Método IV: las ideas. Su hábitat, su vida, sus costumbres, su organización. Madrid: Cátedra.

Morin, E. (1994). Introducción al Pensamiento Complejo. Barcelona: Gedisa.

Morin, E. (2001). La mente bien ordenada. Repensar la reforma. Reformar el pensamiento. Barcelona: Seix Barral.

Naredo, J. M. (2006) Raíces económicas del deterioro ecológico y social: más allá de los dogmas. Madrid: Siglo XXI.

Novo, M. (1998). La Educación Ambiental. Bases éticas, conceptuales y metodológicas. Madrid: Universitas.

Novo, M. (2009). La educación ambiental, una genuina educación para el desarrollo sostenible. Revista de Educación, número extraordinario 2009, 195-217.

ONU (2015). Objetivos desarrollo sostenible. https://www.un.org/sustainabledevelopment/es/objetivos-de-desarrollosostenible/

O’Neill, R. V., De Angelis, D. L., Waide, J. B. y Allen, T. F. H. (1986). A Hierarchical Concept of Ecosystem. Princeton: Princeton University Press.

Prats, S., Herrero, Y. y Torrego, A. (2016). La Gran Encrucijada. Barcelona: Libros en Acción /Icaria.

Riechmann, J. (1995). Desarrollo sostenible; la lucha por la interpretación, en J. Riechmann, J. M. Naredo y otros, De la economía a la ecología, pp. 11-35. Madrid: Trotta

Rodríguez, F. y García, E. (2009). El activismo que no cesa. Obstáculos para incorporar la metodología didáctica basada en la investigación del alumno a la práctica de la Educación Ambiental. Investigación en la Escuela, 67, 23-36.

Rodríguez-Marín, F., Fernández-Arroyo, J. y García Díaz, J. E. (2014). Las Hipótesis de Transición como herramienta didáctica para la Educación Ambiental. Enseñanza de las Ciencias, 32(3), 303-318. 
Rodríguez-Marín, F., Fernández-Arroyo, J. y García-Díaz, J. E. (2015). El huerto escolar ecológico como herramienta para la educación en y para el decrecimiento. Investigación en la Escuela, 86, 35-48.

Rull, V. (2010). El mito del desarrollo sostenible. Collectanea Botanica, 29, 103-109.

Stone, M. K. (2017). Ecoalfabetización y educación para la sostenibilidad. En Educación Ecosocial. Barcelona: Icaria.

Suárez-López, R., Eugenio, M., Lara, F. y Molina-Motos, D. (2018). Examinando el papel de la educación ambiental en la construcción del buen vivir global: contribuciones de la corriente crítica a la definición de objetivos. Iberoamerican Journal of Development Studies, forthcoming. DOI:10.26754/ojs_ried/ijds.338

Taibo, C. (2016). El colapso. Madrid: Los Libros de la Catarata.

Trainer, T. (2017). La vía de la simplicidad. Hacia un mundo sostenible y justo. Madrid: Trotta.

Turiel (2011). Por qué se despilfarra tanto. http://crashoil.blogspot.com.es/2011/09/por-que-se-despilfarra-tanto.html

Turiel, A. (2016). El temor al colapso. http://crashoil.blogspot.com.es/2016/07/eltemor-al-colapso.html

Turiel, A. (2017). De hormigas y hombres. http://crashoil.blogspot.com.es/2017/05/de-hormigas-y-hombres.html

Vilches, A. y Gil, D. (2009). Una situación de emergencia planetaria, a la que debemos y «podemos» hacer frente. Revista de Educación, número extraordinario 2009, 101-122.

Vilches, A., Gil, D. y Cañal, P. (2010). Educación para la sostenibilidad y educación ambiental. Investigación en la Escuela, 71, 5-15. 